\title{
Confronting Einstein Yang Mills Higgs dark energy in light of observations
}

\author{
Debabrata Adak $^{\mathrm{a}}$ (D) \\ Department of Physics, Government General Degree College Singur, Jalaghata Singur, Hooghly, West Bengal 712409, India
}

Received: 15 October 2020 / Accepted: 25 November 2020 / Published online: 4 December 2020

(C) The Author(s) 2020

\begin{abstract}
We study the observational aspects of Einstein Yang Mills Higgs dark energy model and constrain the parameters space from the latest observational data from type Ia supernovae, observational Hubble data, baryon acoustic oscillation data and cosmic microwave background radiation shift parameter data. It is found from the analysis of data that the Higgs field in presence of gauge fields can successfully describe the present accelerated expansion of the universe consistent with the astrophysical observations.
\end{abstract}

\section{Introduction}

Cosmic acceleration discovered more than two decades ago by supernova projects $[1,2]$ is perhaps the most important and fascinating phenomenon that still remains in mystery. This cosmic acceleration can be accounted for by invoking the presence of some exotic fluid dubbed dark energy with negative pressure to overcome the gravitational collapse and thereby resulting in the accelerated expansion of the universe [3-7]. From the observations it is evident that it constitute about $68 \%$ of the total energy density in the universe [8]. The cosmological constant $\Lambda$ is the best fitted model so far to explain this recent accelerated expansion of the universe. However it suffers from two major theoretical problems known as fine tuning problem [9] and cosmic coincidence problem [10]. Despite being consistent with the observations, these problems of cosmological constant make the cosmologists search for alternatives.

A simplest alternative is the canonical scalar field model known as "quintessence" [10]. The potential of the canonical scalar field is so chosen that the field rolls very slowly at the present epoch resulting in the negative pressure of the field which leads to cosmic acceleration. This essentially requires the potential to be very flat with respect to the field $\phi$ resulting in the mass of the field around $10^{-33} \mathrm{eV}$. The tracker

a e-mail: debabrata.adak.sinp@gmail.com (corresponding author) behaviour of the scalar field model [11] helps to alleviate the problem of cosmic coincidence in the dark energy scenario. Explaining the late time cosmic acceleration is also possible from the modification of gravity at the large scales known as infrared modification of gravity. It is found that higher order curvature invariants play an important role in modification of gravity at large scales thereby leading to accelerated expansion of the universe at the present epoch [12-14]. Moreover higher dimensional models of gravity induces modification of Einstein's gravity in the $3+1$ dimensional effective theory at large scales leading to the accelerated expansion of the universe $[15,16]$. A large number of modified gravity models is tested to be free from ghost or tachyon instabilities and they also do not conflict with the solar system constraints (see $[17,18]$ and references therein for a review on cosmology driven by modified gravity models). Of late the detections of GW170817 and GRB 170817A revealed the fact that the speed of gravitational waves differs from the speed of light by one part in $10^{15}$ [19-23]. This discovery have severely constrained these modified gravity models as well as other dark energy models [24-32].

From the standard model of particle physics this is well known that all the particles in the universe get masses due to their interaction with the Higgs field $[33,34]$. The dynamics of FRW universe was studied in presence of non-abelian gauge fields invariant under $\mathrm{SO}(3)$ and $\mathrm{SU}(2)$ gauge group or an arbitrary gauge group $\mathrm{SO}(\mathrm{N})$ [35-42]. In the context of inflation Einstein Yang-Mills Higgs action was first introduced $[43,44]$ to study the effect of gauge field on inflation. There are also models of inflation known as Gaugeflation where the inflation is driven solely by a non-Abelian gauge field, see for instance Refs. [45-47]. In these models the Yang-Mills gauge sector is modified to include a specific higher order derivative operator for driving inflationary dynamics whereas in the other works [40-42], the Yang Mills gauge sector is non-minimally coupled to gravity or associated with some potential for the same reason. In the case 
of standard model Higgs field no such thing is there [48]. Recently in [49], the dynamics of this non-abelian Higgs field coupled to gravity was studied in the context of late time cosmic acceleration. In the work [50], considering the interaction in $\mathrm{SU}(2)$ representation for Higgs field the authors have studied the dynamics of cosmology in Einstein YangMills Higgs to explain the recent accelerated expansion of the Universe. The main motivation behind considering the Yang Mills gauge field with the Higgs field lies in the Mexican hat potential of the Higgs field [49]. The Mexican hat potential of the Higgs field is steep enough to sustain the late time cosmic acceleration without a very fine tuned initial condition. The presence of Yang Mills gauge field in the standard model of particles helps to alleviate the fine tuning of the initial conditions by increasing the Hubble friction and flattening the Higgs potential as we shall see in next section. Moreover what is not yet known is that the viability of this model in respect of cosmological observations.

In the present work we study the viability of the Einstein Yang-Mills Higgs dark energy in the context of observational data. We constrain the model from type Ia supernova data (SNe Ia), observational Hubble data (OHD), baryon acoustic oscillation data (BAO) and cosmic microwave background shift parameter data (CMB) and show that the model parameter space is consistent with the cosmological observations thus making this a viable model for dark energy to explain the current accelerated expansion of the universe. This paper is organised as follows. In Sect. 2 we review the Einstein Yang-Mills Higgs action coupled to gravity and the equations of motion in FRW background to study the dynamical system. Construction of autonomous system and dynamics of cosmology is studied in Sect. 3. In Sect. 4 we discuss the various observational data and the formalism for analyses of those data. In this section we also confront this Higgs dark energy model with the observational data and present the results of our data analysis. Eventually we conclude in Sect. 5.

\section{Einstein Yang-Mills Higgs action}

In what follows we describe the Higgs dark energy in presence of gauge field in background of Einstein's gravitation. The Einstein Yang-Mills Higgs action is given by [49,50],

$$
\begin{aligned}
S=\int & d^{4} x \sqrt{-g}\left(\frac{M_{\mathrm{Pl}}^{2}}{2} R-\frac{1}{4} F_{a}^{\mu \nu} F_{\mu \nu}^{a}-\left(D^{\mu} \Phi\right)^{\dagger}\left(D_{\mu} \Phi\right)\right. \\
& \left.-V(\Phi)+L_{r}+L_{m}\right)
\end{aligned}
$$

where $M_{\mathrm{Pl}}$ is the reduced Planck mass given by $M_{\mathrm{Pl}}=$ $1 / \sqrt{8 \pi G}, g$ is the determinant of spacetime metric, $\Phi$ is the complex Higgs doublet invariant under SU(2) gauge symme- try, $L_{r}$ is the lagrangian for radiation and $L_{m}$ is the matter lagrangian. Here $F_{\mu \nu}^{a}$ is the rank-2 tensor that represents the non-Abelian gauge field and is given by

$F_{\mu \nu}^{a}=\partial_{\mu} A_{\nu}^{a}-\partial_{\nu} A_{\mu}^{a}+\beta \epsilon_{b c}^{a} A_{\mu}^{b} A_{\nu}^{c}$,

where $A_{\mu}^{a}$ is the gauge field, $\beta$ is the coupling of SU(2) group and $\epsilon_{b c}^{a}$ is the rank 3 Levi-Civita symbol. $D_{\mu}$ is the gauge covariant derivative given by

$D_{\mu}=\nabla_{\mu}-i \beta \frac{\sigma_{a}}{2} A_{\mu}^{a}$,

where $\nabla_{\mu}$ is the spacetime covariant derivative and $\sigma_{a}$ are the Pauli matrices. The complex Higgs doublet and its potential are respectively given by,

$\Phi=\left(\begin{array}{l}\phi_{1}+i \chi_{1} \\ \phi_{2}+i \chi_{2}\end{array}\right)$

where $\phi_{1}, \phi_{2}, \chi_{1}, \chi_{2}$ are real scalar fields and

$V(\Phi)=\frac{\lambda}{4}\left(\Phi^{\dagger} \Phi-v^{2}\right)^{2}$,

where $v$ is the vacuum expectation value (VEV) of Higgs field.

It is evident from the observations [8] that our universe is homogeneous and isotropic on large scales. Hence the background spacetime of the universe is described by the Friedmann Lemaître Robertson Walker (FLRW) metric and is given by in spherically symmetric coordinates,

$d s^{2}=-d t^{2}+a^{2}(t)\left(d r^{2}+r^{2} d \Omega^{2}\right)$,

where $t$ is the cosmological time, $a(t)$ is scale factor for expanding universe and $d \Omega^{2}=d \theta^{2}+\sin ^{2} \theta d \phi^{2}$. The energy momentum tensor for the action in Eq. 1 is given by,

$$
\begin{aligned}
& T_{\mu \nu}=-F_{\mu \eta}^{a} F_{\nu a}^{\eta}-\left(D_{\mu} \Phi\right)^{\dagger}\left(D_{\nu} \Phi\right)-\left(D_{\nu} \Phi\right)^{\dagger}\left(D_{\mu} \Phi\right) \\
& +2 \frac{\partial}{\partial g^{\mu \nu}}\left(L_{m}+L_{r}\right) \\
& -g_{\mu \nu}\left[-\frac{1}{4} F_{a}^{\mu \nu} F_{\mu \nu}^{a}-\left(D^{\mu} \Phi\right)^{\dagger}\left(D_{\mu} \Phi\right)-V(\Phi)+L_{r}+L_{m}\right] .
\end{aligned}
$$

The Einstein tensor $G_{\mu \nu}$ is diagonal for FLRW background spacetime and hence the off diagonal terms of energy momentum tensor should vanish. This condition makes the gauge field become $A_{\mu}^{a}=\delta_{\mu}^{a} f(t)$ [49] where $f(t)$ is the only degree of freedom in the gauge sector as allowed from the FLRW spacetime of the universe, $a$ is the gauge index and $i$ is the spatial index. As discussed in [50], this condition is not sufficient to avoid the non-zero contribution to the momentum density arising from the interaction between the Yang-Mills field and the Higgs field. Hence another additional condition which is required to establish the isotropy in energy momentum tensor is to fix the gauge so that

$$
\Phi(t)=\left(\begin{array}{c}
\phi(t) \\
0
\end{array}\right)
$$


where $\phi(t)$ is a real scalar field. With these choices of gauge field and gauge fixation for the Higgs field, we obtain

$$
\begin{gathered}
H^{2}=\frac{1}{3 M_{\mathrm{Pl}}^{2}}\left[\frac{3}{2} \frac{\dot{f}(t)^{2}}{a(t)^{2}}+\dot{\phi}(t)^{2}+\frac{3}{2} \frac{\beta^{2} f(t)^{4}}{a(t)^{4}}\right. \\
\left.+\frac{3}{4} \frac{\beta^{2} \phi(t)^{2} f(t)^{2}}{a(t)^{2}}+V(\phi)+\rho_{m}+\rho_{r}\right], \\
\dot{H}=-\frac{1}{2 M_{\mathrm{Pl}}^{2}}\left[2 \frac{\dot{f}(t)^{2}}{a(t)^{2}}+2 \dot{\phi}(t)^{2}+2 \frac{\beta^{2} f(t)^{4}}{a(t)^{4}}\right. \\
\left.+\frac{\beta^{2} \phi(t)^{2} f(t)^{2}}{2 a(t)^{2}}+\rho_{m}+\frac{4}{3} \rho_{r}\right],
\end{gathered}
$$

where $H$ is Hubble parameter given by $H=\dot{a}(t) / a(t)$ and $\rho_{m}, \rho_{r}$ are the matter and radiation density respectively. The equation of motions of the gauge and Higgs fields are respectively given by,

$$
\begin{gathered}
\ddot{f}(t)+H \dot{f}(t)+\beta^{2}\left[2 \frac{f(t)^{3}}{a(t)^{2}}+\frac{f(t) \phi(t)^{2}}{2}\right]=0, \\
\ddot{\phi}(t)+3 H \dot{\phi}(t)+\frac{3 \beta^{2} f(t)^{2} \phi(t)}{4 a(t)^{2}}+\frac{d V(\phi)}{d \phi}=0,
\end{gathered}
$$

where $V(\phi)$ is given by $V(\phi)=\frac{\lambda}{4}\left(\phi^{2}-v^{2}\right)$. This is worth mentioning here that from Eq. (11) it is evident that there arises an effective potential for the gauge field with vanishing vacuum expectation value due to the interaction between the gauge field and the Higgs field. Moreover the same interaction leads to the effective potential of the Higgs field also as shown in the Eq. (12).

\section{Dynamics of cosmology}

To analyse the cosmological dynamics the following dimensionless variables are introduced here.

$$
\begin{aligned}
& x_{1}=\frac{\dot{f}}{\sqrt{2} a M_{\mathrm{Pl}} H}, \quad y_{1}=\frac{\beta f^{2}}{\sqrt{2} a^{2} M_{\mathrm{Pl}} H}, \\
& z_{1}=\frac{\beta f \phi}{2 a M_{\mathrm{Pl}} H}, \quad x_{2}=\frac{\dot{\phi}}{\sqrt{3} M_{\mathrm{Pl}} H}, \\
& y_{2}=\sqrt{\frac{V(\phi)}{3 M_{\mathrm{Pl}}^{2} H^{2}}}, \quad r=\sqrt{\frac{\rho_{r}}{3 M_{\mathrm{Pl}}^{2} H^{2}}}, \\
& m=\sqrt{\frac{\rho_{m}}{3 M_{\mathrm{Pl}}^{2} H^{2}}}, \quad w_{1}=\frac{\sqrt{2} a M_{\mathrm{Pl}}}{f} .
\end{aligned}
$$

The subscripts 1 and 2 refers to the dimensionless variables corresponding to gauge field and the Higgs field. With these choices the total energy density in the universe takes the form (from Eq. (10)),

$$
x_{1}^{2}+y_{1}^{2}+z_{1}^{2}+x_{2}^{2}+y_{2}^{2}+r^{2}+m^{2}=1 \text {. }
$$

The evolution equations of the autonomous system are given by

$$
\begin{aligned}
x_{1}^{\prime} & =x_{1}(q-1)-w_{1}\left(2 y_{1}^{2}+z_{1}^{2}\right), \\
y_{1}^{\prime} & =y_{1}\left(2 x_{1} w_{1}+q-1\right), \\
z_{1}^{\prime} & =z_{1}\left(x_{1} w_{1}+q\right)+\frac{\sqrt{3}}{2} w_{1} y_{1} x_{2}, \\
x_{2}^{\prime} & =x_{2}(q-2)-z_{1} w_{1}\left(2 \alpha y_{2}+\frac{\sqrt{3}}{2} y_{1}\right), \\
y_{2}^{\prime} & =y_{2}(q+1)+\alpha w_{1} z_{1} x_{2}, \\
r^{\prime} & =r(q-1), \\
m^{\prime} & =m\left(q-\frac{1}{2}\right), \\
w_{1}^{\prime} & =w_{1}\left(1-w_{1} x_{1}\right),
\end{aligned}
$$

where the symbol prime denotes a derivative with respect to $N=\ln a, a$ being the scale factor of the universe and $q$ is the deceleration parameter defined as $q(t)=-\frac{\ddot{a}(t) a(t)}{\dot{a}(t)^{2}}$. In terms of the dimensionless variables defined above the deceleration parameter takes the form

$q=\frac{1}{2}\left(1+x_{1}^{2}+y_{1}^{2}-z_{1}^{2}+3 x_{2}^{2}-3 y_{2}^{2}+r^{2}\right)$.

Here $\alpha$ is a dimensionless constant given by $\alpha=\sqrt{\frac{\lambda}{2 \beta^{2}}}$.

The dynamical analysis of the model reveals that there exists an attractor solution for the model in $x_{1}=0, y_{1}=$ $0, z_{1}=0, x_{2}=0, y_{2}=1, r=0, m=0, w_{1}=0$ plane for which the deceleration parameter turns out to be $q=-1$. This attractor solution corresponds to late-time cosmic acceleration. In Fig. 1, the dynamical evolution of the model is shown in the phase planes $\left(x_{1}, y_{1}\right)$ and $\left(x_{1}, y_{2}\right)$.

In the Fig. 1 (left) the system starts from $(1,0)$ (solid red), $(1,1)$ (red dotdashed), $(1,2)$ (red dashed), $(0.3,2)$ (solid blue), $(0,2)$ (blue dashed), $(-0.3,2)$ (blue dotdashed) $(-1,2)$ (green dotdashed), $(-1,1)$ (green dashed) and $(-1,0)$ (solid green $)$ in the $\left(x_{1}, y_{1}\right)$ plane and approaches the attractor solution $(0,0)$ as $N$ changes from $N=-15$ to $N=0$. Similarly, in the Fig. 1 (right) the system starts from $(1,1)$ (solid red), (1, 2) (red dashed), $(1,5)$ (red dotdashed), $(0.5,5)$ (solid blue), $(0,5)$ (blue dashed), $(-0.5,5)$ (blue dotdashed), $(-1,5)$ (green dashed), $(-1,2)$ (green dotdashed) and $(-1,1)$ (solid green $)$ in the $\left(x_{1}, y_{2}\right)$ plane and approaches the attractor solution $(0,1)$ as $N$ changes from $N=-15$ to $N=0$. We solve the autonomous system for the initial conditions given by $x_{1}=10^{-18}, y_{1}=10^{-18}, z_{1}=10^{-18}, x_{2}=10^{-18}$, $y_{2}=0.831, w_{1}=10^{2}$ and $r=10^{-2}$ at $z=0$. These initial conditions chosen here, are in consideration with the Higgs vev $(v \sim 246 \mathrm{GeV})$, radiation density parameter at the present epoch of order $10^{-4}$ and the total length of the radiation dominated epoch (see $[49,50]$ for details). The velocities 


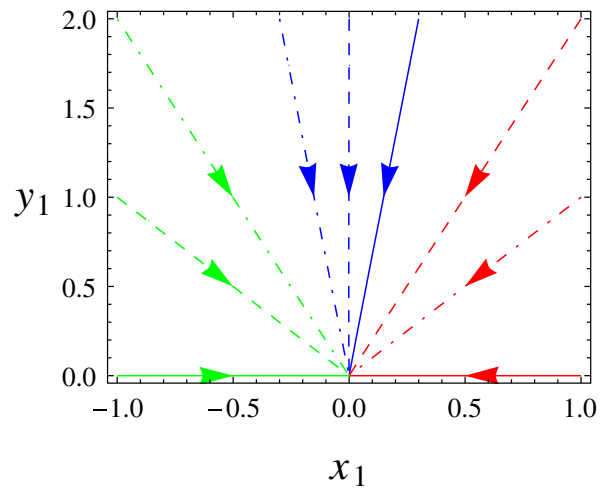

Fig. 1 Evolution of the three dimensionless parameters $x_{1}, y_{1}$ and $y_{2}$ are shown in the $\left(x_{1}, y_{1}\right)$ and $\left(x_{1}, y_{2}\right)$ plane. In the left figure the system starts from $(1,0)$ (solid red), $(1,1)$ (red dotdashed), $(1,2)$ (red dashed), $(0.3,2)$ (solid blue), $(0,2)$ (blue dashed), $(-0.3,2)$ (blue dotdashed) $(-1,2)$ (green dotdashed), $(-1,1)$ (green dashed) and $(-1,0)$ (solid green $)$ in the $\left(x_{1}, y_{1}\right)$ plane and approaches the attractor solution $(0,0)$

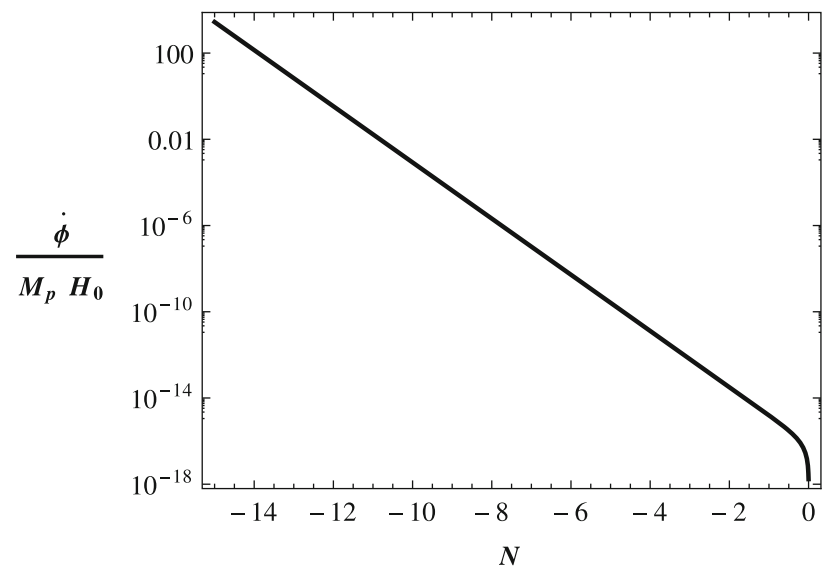

Fig. 2 Velocity of Higgs field $\frac{\dot{\phi}}{M_{P} H_{0}}$ plotted against the number of e-foldings $N$

of the Higgs field and the gauge field are shown in the Figs. 2 and 3 .

From the Figs. 2 and 3, it is evident that the Higgs and the gauge field are moving very slowly. The reason behind this is the presence of gauge field which introduces enough Hubble friction as well as flattens the Mexican hat potential of Higgs field (Eq. 12) so as to make the Higgs field move very slowly along the potential. Later this fact will also be reflected in the Fig. 4 (top) where $\rho_{\mathrm{DE}}$ starts mimicking cosmological constant deep in the radiation era. In the Fig. 4 (top), we show the variation of density of radiation, matter and dark energy with the number of e-foldings $N$ and variation of the density parameters are shown in Fig. 5 for $\Omega_{m}^{(0)}=0.31$ and $H_{0}=69 \mathrm{~km} \mathrm{~s}^{-1} \mathrm{Mpc}^{-1}$ and $\alpha=1$. From these two figures this is evident that the dark energy dominates very recently (around $N \sim-0.25$ as clearly shown in Fig. 4 (bottom)

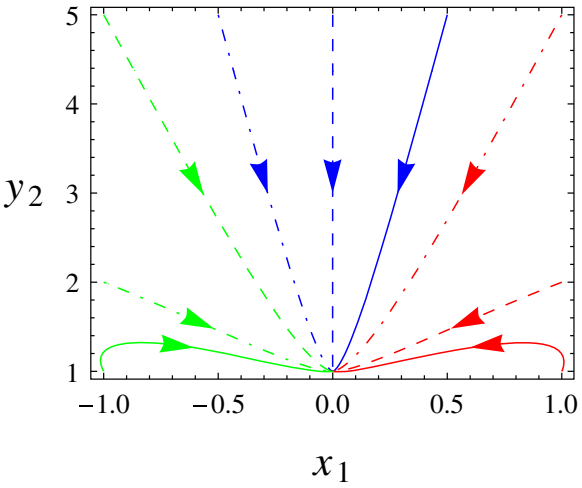

as $N$ changes from $N=-15$ to $N=0$. In the right figure the system starts from $(1,1)$ (solid red), $(1,2)$ (red dashed), $(1,5)$ (red dotdashed), $(0.5,5)$ (solid blue), $(0,5)$ (blue dashed), $(-0.5,5)$ (blue dotdashed), $(-1,5)$ (green dashed), $(-1,2)$ (green dotdashed) and $(-1,1)$ (solid green) in the $\left(x_{1}, y_{2}\right)$ plane and approaches the attractor solution $(0,1)$ as $N$ changes from $N=-15$ to $N=0$

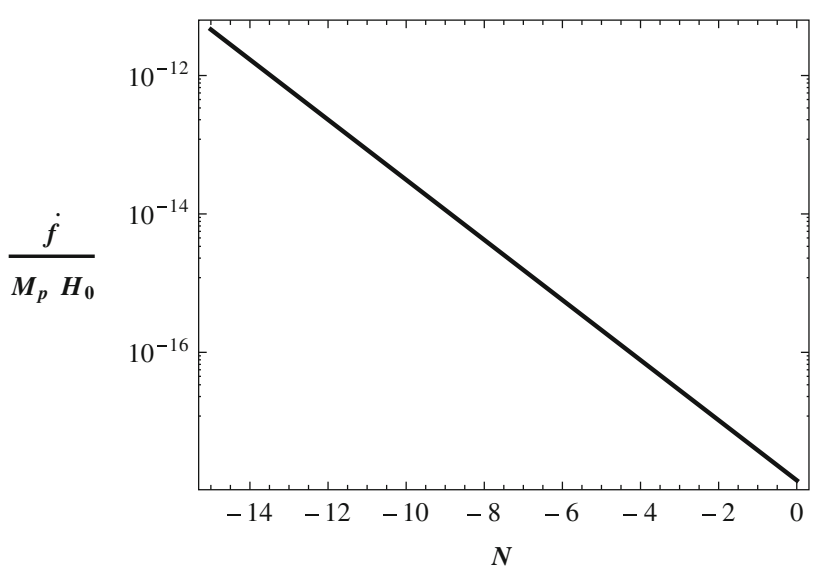

Fig. 3 Velocity of gauge field $\frac{\dot{f}}{M_{P} H_{0}}$ plotted against the number of e-foldings $N$

also). Moreover it is evident from Fig. 4 (top) that the Higgs dark energy though varies initially but starts mimicking the cosmological constant around $N=-12$ i.e., well in the radiation dominated era. The plot of the deceleration parameter $q$ and the effective equation of state $\omega_{\text {eff }}$ of the universe for all the components i.e., radiation, matter and the dark energy are shown in Fig. 6. The acceleration of the universe corresponds to $q<0$ and $\omega_{\text {eff }}<-1 / 3$.

\section{Observational constraints}

In this era of precision cosmology models of dark energy are highly constrained. Passing the test of observational data only makes the model acceptable despite their theoretical viability. In this section we describe the observational data that are used to constrain the model parameters in this Einstein Yang-Mills 

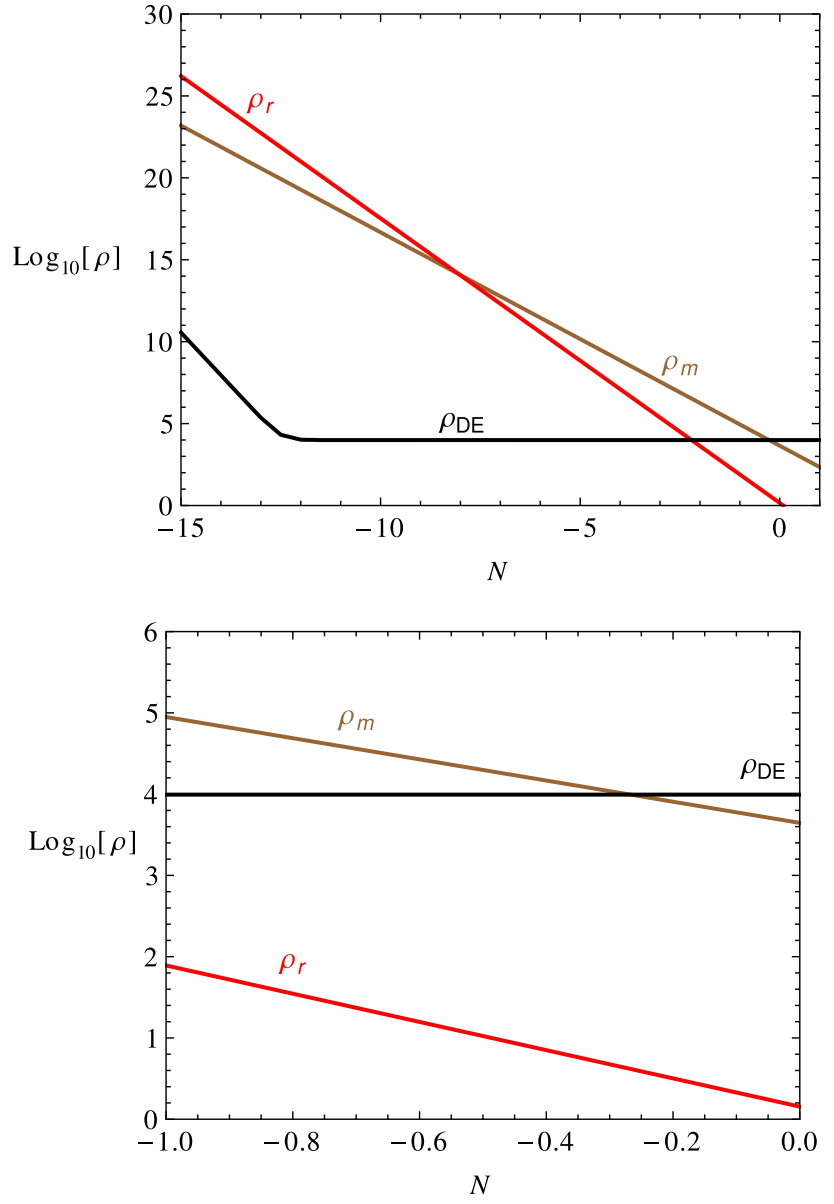

Fig. 4 Plot of the density in the Universe with number of e-foldings $N$ given by $N=-\ln (1+z)$ where $z$ is the corresponding redshift

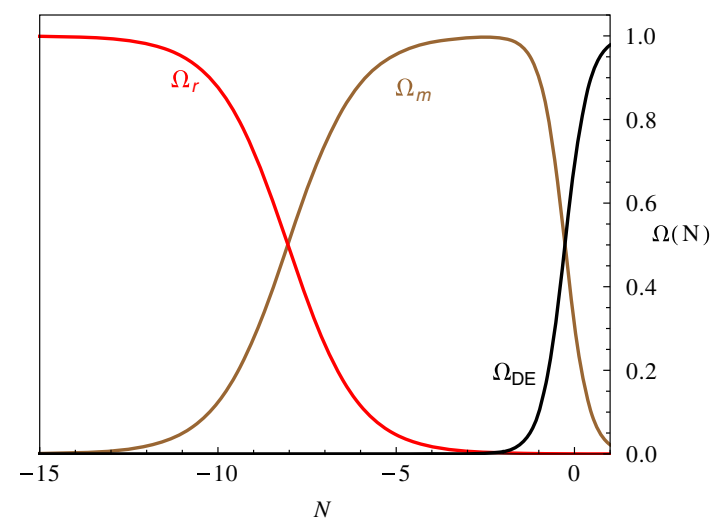

Fig. 5 Plot of the density parameters in the Universe with the number of e-foldings $N$ given by $N=-\ln (1+z)$ where $z$ is the corresponding redshift

Higgs dark energy and the formalism for the data analysis as well.

Supernovae Type Ia are accepted as the standard candles in astrophysical observations. Incidentally it happened to be the first probe for the discovery of the late time cosmic acceleration $[1,2]$. We consider here 279 Supernovae Type Ia (SNe

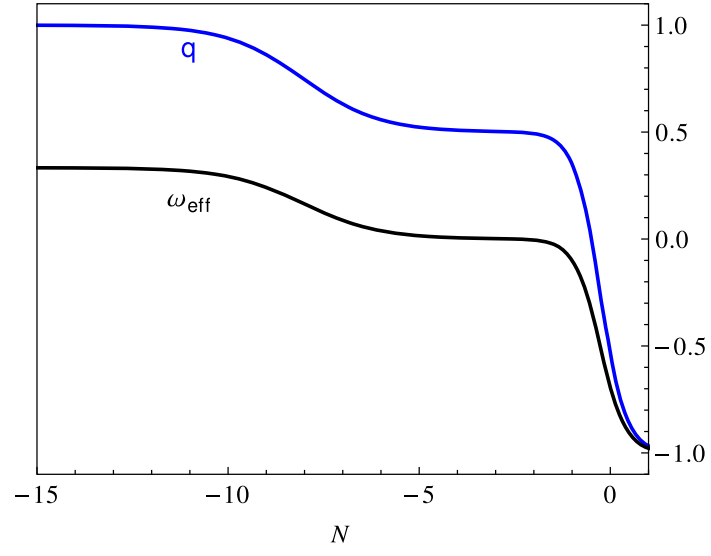

Fig. 6 Plot of deceleration parameter $q$ and effective equation of state of the universe $\omega_{\mathrm{eff}}$ with the number of e-foldings $N$ given by $N=$ $-\ln (1+z)$ where $z$ is the corresponding redshift

Ia) observational data from Pan-STARRS1 Medium Deep Survey in the redshift range $0.03<z<0.68$ along with the other SNe Ia data from Sloan Digital Sky Survey (SDSS) [51-53], SNLS [54,55], and ESSENCE [56-58] and SCP [59]. The combined data set known as Pantheon Sample [60] consists of $1048 \mathrm{SNe}$ Ia data points in the redshift range $0.01<z<2.3$. The distance modulus for type Ia supernova as a function of the redshift is given by

$\mu(z)=5 \log _{10}\left(D_{L}(z)\right)+\mu_{0}$,

where $D_{L}(z)=H_{0} d_{L}(z) / c$ ( $c$ is speed of light in free space) and $\mu_{0}=42.38-5 \log _{10} h$ for $H_{0}=100 h \mathrm{~km} \mathrm{~s}^{-1} \mathrm{Mpc}^{-1}$. The chi-square for supernovae data is defined as

$\chi_{\mathrm{SN}}^{2}\left(p_{s}\right)=\sum_{i}\left[\frac{\mu_{\mathrm{obs}}\left(z_{i}\right)-\mu_{\mathrm{th}}\left(z_{i}, p_{s}, \mu_{0}\right)}{\sigma_{i}}\right]^{2}$,

where $p_{s}$ are the model parameters and $z_{i}$ are the redshifts of the observational supernovae type Ia data. $\mu_{\text {obs }}$ and $\mu_{\text {th }}$ are the observational and theoretical distance modulus respectively. The chi-square is marginalised over the nuisance parameter $\mu_{0}[61]$ and the marginalised chi-square is given by

$\chi_{\mathrm{SN}}^{2}=A\left(p_{s}\right)-\frac{B\left(p_{s}\right)^{2}}{C}$,

where $A, B$ and $C$ are given by

$$
\begin{aligned}
A\left(p_{s}\right) & =\sum_{i}\left[\frac{\mu_{\mathrm{obs}}\left(z_{i}\right)-\mu_{\mathrm{th}}\left(z_{i}, p_{s}, \mu_{0}\right)}{\sigma_{i}}\right]^{2}, \\
B\left(p_{s}\right) & =\sum_{i} \frac{\mu_{\mathrm{obs}}\left(z_{i}\right)-\mu_{\mathrm{th}}\left(z_{i}, p_{s}, \mu_{0}\right)}{\sigma_{i}^{2}}, \\
C & =\sum_{i} \frac{1}{\sigma_{i}^{2}} .
\end{aligned}
$$


Cosmic microwave background shift parameter $R$ is a model independent parameter that can also be used to constrain the models of dark energy. It is obtained from the first peak of temperature anisotropy plot of the cosmic microwave background radiation. The CMBR shift parameter is defined as

$R\left(z_{*}\right)=\left(\Omega_{m}^{0} H_{0}^{2}\right)^{1 / 2} \int_{0}^{z_{*}} \frac{d z}{H(z)}$,

where $z_{*}$ corresponds to the redshift of the radiation matter decoupling epoch. The chi-square for CMBR shift parameter is defined as

$\chi_{\mathrm{CMB}}^{2}=\left[\frac{R_{\mathrm{th}}\left(z_{*}, p_{s}\right)-R_{\mathrm{obs}}\left(z_{*}\right)}{\sigma_{R}}\right]^{2}$.

Needless to mention that $p_{s}$ are the model parameters. We use the CMBR shift parameter from latest Planck observations $R=1.7499 \pm 0.0088$ at the redshift of decoupling era $z_{*}=1091.41[62]$.

Observational Hubble data is a direct measurement of expansion rate of universe with the redshifts. It is another tool to constrain the dark energy models. The chi-square for observational Hubble data is given by

$\chi_{\mathrm{OHD}}^{2}=\sum_{i}\left[\frac{H_{\mathrm{obs}}\left(z_{i}\right)-H_{\mathrm{th}}\left(z_{i}, p_{s}\right)}{\sigma_{i}^{2}}\right]^{2}$.

We use the 31 data points of $H(z)$ for the purpose of $\chi_{\mathrm{OHD}}^{2}$ analysis.

$\boldsymbol{C}=\left(\begin{array}{llllllll}0.0150 & -0.0357 & 0.0071 & -0.0100 & 0.0032 & -0.0036 & 0 & 0 \\ -0.0357 & 0.5304 & -0.0160 & 0.1766 & -0.0083 & 0.0616 & 0 & 0 \\ 0.0071 & -0.0160 & 0.0182 & -0.0323 & 0.0097 & -0.0131 & 0 & 0 \\ -0.0100 & 0.1766 & -0.0323 & 0.3267 & -0.0167 & 0.1450 & 0 & 0 \\ 0.0032 & -0.0083 & 0.0097 & -0.0167 & 0.0243 & -0.0352 & 0 & 0 \\ -0.0036 & 0.0616 & -0.0131 & 0.1450 & -0.0352 & 0.2684 & 0 & 0 \\ 0 & 0 & 0 & 0 & 0 & 0 & 0.1358 & -0.0296 \\ 0 & 0 & 0 & 0 & 0 & 0 & -0.0296 & 0.0492\end{array}\right)$.

The measurements of observational Hubble data are summarized in Table 1 [70].

Before the recombination epoch the baryons were tightly coupled to the photons and as a result of this tight coupling the acoustic oscillations created small density fluctuations in baryon photon plasma. In the expanding universe, this density fluctuations left an imprint in the large scale structures which provides a standard ruler in cosmology. Baryon acoustic oscillation is the powerful tool for constraining dark energy models. The sound horizon at a redshift $z_{d}$ for drag data is given by

$\chi_{\mathrm{BAO}}^{2}=\chi_{\text {iso }}^{2}+\chi_{\text {aniso }}^{2}$,

where epoch is given by

$r_{d}=\frac{c}{\sqrt{3}} \int_{z_{d}}^{\infty} \frac{d z}{\sqrt{1+\frac{3 \Omega_{b}^{(0)}}{4 \Omega_{\gamma}^{(0)}} \frac{1}{1+z}} H(z)}$,

where the drag redshift $z_{d}$ is given by

$z_{d}=\frac{1291\left(\Omega_{m}^{(0)} h^{2}\right)^{0.251}}{1+0.659\left(\Omega_{m}^{(0)} h^{2}\right)^{0.828}}\left[1+b_{1}\left(\Omega_{b}^{(0)} h^{2}\right)^{b_{2}}\right]$,

with

$b_{1}=0.313\left(\Omega_{b}^{(0)} h^{2}\right)^{-0.419}\left[1+0.607\left(\Omega_{m}^{(0)} h^{2}\right)^{0.674}\right]$,

$b_{2}=0.238\left(\Omega_{m}^{(0)} h^{2}\right)^{0.223}$,

and $\Omega_{b}^{(0)} h^{2}=0.02236, \Omega_{\gamma}^{(0)} h^{2}=2.469 \times 10^{-5}$ [8]. In a spatially flat universe the angular diameter distance $D_{A}(z)$, the Hubble distance $D_{H}(z)$ and the effective distance $D_{V}(z)$ are respectively given by,

$D_{A}(z)=\frac{c}{(1+z)} \int_{0}^{z} \frac{d z}{H(z)}$

$D_{H}(z)=\frac{c}{H(z)}$,

$D_{V}(z)=\left[\left(\frac{d_{L}(z)}{1+z}\right)^{2} \frac{c z}{H(z)}\right]^{1 / 3}$

where $c$ the speed of light in vacuum. Here we use both the isotropic and anisotropic BAO data that are tabulated in Tables 2 and 3 [71].

The covariance matrix $\boldsymbol{C}$ associated with the anisotropic BAO measurements is given by

The total chi-square for isotropic and anisotropic BAO

$$
\chi_{\mathrm{iso}}^{2}=\sum_{i}\left[\frac{D_{V}\left(z_{i}\right) / r_{d}-D_{V}\left(z_{i}, p_{s}\right) / r_{d}}{\sigma_{i}}\right]^{2},
$$


Table 1 The $31 H(z)$ data points [70]

\begin{tabular}{|c|c|c|}
\hline$z$ & $\begin{array}{l}H(z) \\
\mathrm{km} \mathrm{s}^{-1} \mathrm{Mpc}^{-1}\end{array}$ & $\begin{array}{l}\sigma_{H(z)} \\
\mathrm{km} \mathrm{s}^{-1} \mathrm{Mpc}^{-1}\end{array}$ \\
\hline 0.07 & 69.0 & $19.6[63]$ \\
\hline 0.09 & 69.0 & $12.0[64]$ \\
\hline 0.12 & 68.6 & $26.2[63]$ \\
\hline 0.17 & 83.0 & 8.0 [64] \\
\hline 0.179 & 75.0 & $4.0[65]$ \\
\hline 0.199 & 75.0 & $5.0[65]$ \\
\hline 0.2 & 72.9 & $29.6[63]$ \\
\hline 0.27 & 77.0 & $14.0[64]$ \\
\hline 0.28 & 88.8 & $36.6[63]$ \\
\hline 0.352 & 83.0 & $14.0[65]$ \\
\hline 0.3802 & 83.0 & $13.5[66]$ \\
\hline 0.4 & 95.0 & $17.0[64]$ \\
\hline 0.4004 & 77.0 & $10.2[66]$ \\
\hline 0.4247 & 87.1 & $11.2[66]$ \\
\hline 0.4497 & 92.8 & $12.9[66]$ \\
\hline 0.47 & 89.0 & 49.6 [67] \\
\hline 0.4783 & 80.9 & 9.0 [66] \\
\hline 0.48 & 97.0 & $62.0[68]$ \\
\hline 0.593 & 104.0 & $13.0[65]$ \\
\hline 0.68 & 92.0 & $8.0[65]$ \\
\hline 0.781 & 105.0 & $12.0[65]$ \\
\hline 0.875 & 125.0 & $17.0[65]$ \\
\hline 0.88 & 90.0 & $40.0[68]$ \\
\hline 0.9 & 117.0 & 23.0 [64] \\
\hline 1.037 & 154.0 & $20.0[65]$ \\
\hline 1.3 & 168.0 & $17.0[64]$ \\
\hline 1.363 & 160.0 & 33.6 [69] \\
\hline 1.43 & 177.0 & $18.0[64]$ \\
\hline 1.53 & 140.0 & $14.0[64]$ \\
\hline 1.75 & 202.0 & $40.0[64]$ \\
\hline 1.965 & 186.5 & 50.4 [69] \\
\hline
\end{tabular}

$\chi_{\text {aniso }}^{2}=X_{\text {aniso }}^{T} C^{-1} X_{\text {aniso }}$

where $X_{\text {aniso }}$ is column matrix given by,

$$
X_{\text {aniso }}=\left(\begin{array}{c}
\frac{D_{A}(0.38)}{r_{d}}-7.42 \\
\frac{D_{H}(0.38)}{r_{d}}-24.97 \\
\frac{D_{A}(0.51)}{r_{d}}-8.85 \\
\frac{D_{H}(0.51)}{r_{d}}-22.31 \\
\frac{D_{A}(0.61)}{r_{d}}-9.69 \\
\frac{D_{H}(0.61)}{r_{d}}-20.49 \\
\frac{D_{A}(2.4)}{r_{d}}-10.76 \\
\frac{D_{H}(2.4)}{r_{d}}-8.94
\end{array}\right) .
$$

Table 2 Isotropic BAO data

\begin{tabular}{lll}
\hline Data set & Redshift & $D_{V}(z) / r_{d}$ \\
\hline $6 \mathrm{dF}$ & $\mathrm{z}=0.106$ & $2.98 \pm 0.13[72]$ \\
MGS & $\mathrm{z}=0.15$ & $4.47 \pm 0.17[73]$ \\
eBOSS quasars & $\mathrm{z}=1.52$ & $26.1 \pm 1.1[74]$ \\
\hline
\end{tabular}

Table 3 Anisotropic BAO data

\begin{tabular}{lll}
\hline Data set & Redshift & $D_{A / H}(z) / r_{d}$ \\
\hline BOSS DR12 & $\mathrm{z}=0.38$ & $7.42(A)[75]$ \\
BOSS DR12 & $\mathrm{z}=0.38$ & $24.97(H)[75]$ \\
BOSS DR12 & $\mathrm{z}=0.51$ & $8.85(A)[75]$ \\
BOSS DR12 & $\mathrm{z}=0.51$ & $22.31(H)[75]$ \\
BOSS DR12 & $\mathrm{z}=0.61$ & $9.69(A)[75]$ \\
BOSS DR12 & $\mathrm{z}=0.61$ & $20.49(H)[75]$ \\
BOSS DR12 & $\mathrm{z}=2.4$ & $10.76(A)[76]$ \\
BOSS DR12 & $\mathrm{z}=2.4$ & $8.94(H)[76]$ \\
\hline
\end{tabular}

The total combined chi-square for all the aforesaid data sets i.e., $\mathrm{SNe}$ Ia, CMB shift parameter, OHD, BAO is given by,

$\chi_{\mathrm{tot}}^{2}=\chi_{\mathrm{SN}}^{2}+\chi_{\mathrm{OHD}}^{2}+\chi_{\mathrm{BAO}}^{2}+\chi_{\mathrm{CMB}}^{2}$.

We use this total chisquare defined in Eq. (37) for the data analysis purpose of the Yang-Mills Higgs dark energy model and constrain the parameters space.

In what follows, we describe the model parameters and the results of the chi-square analysis of the observational data. In this Higgs dark energy model we consider four parameters namely $\alpha, \Omega_{m}^{(0)}, H_{0}$ and $H_{0} r_{d} / c$ to fit the chi-square with the latest observational data from $\mathrm{SNe} \mathrm{Ia}, \mathrm{OHD}, \mathrm{BAO}$ and $\mathrm{CMB}$. In the Fig. 7, we present the 68.3, 90 and $99 \%$ confidence level plot for the parameters $\Omega_{m}^{(0)}$ and $H_{0}$ with the contour shadding by the light blue, dark blue and cyan colours respectively. The total chi-square turns out to have a minima at $\Omega_{m}^{(0)} \simeq 0.315$ and $H_{0} \simeq 68.6 \mathrm{~km} \mathrm{~s}^{-1} \mathrm{Mpc}^{-1}$ and $H_{0} r_{d} / c \simeq 0.0335$ which with the best-fit value of $H_{0}$ and speed of light in vacuum gives $r_{d} \simeq 146.5 \mathrm{Mpc}$.

Figures 8 and 9 shows the observationally allowed parameters space in $\Omega_{m}^{(0)}-H_{0} r_{d} / c$ and $H_{0} r_{d} / c-H_{0}$ at 68.3, 90 and $99 \%$ confidence levels with the same colours mentioned above.

It is worth mentioning here that all this confidence contours corresponds to value of $\alpha=1$ [77]. The parameter $\alpha$ cannot be constrained from the present observational data we have considered here. A confidence contour is shown in Fig. 10 in the $\alpha-\Omega_{m}^{(0)}$ parameters space from where it is evident that the present data is unable to put any bound on the parameter $\alpha$. 


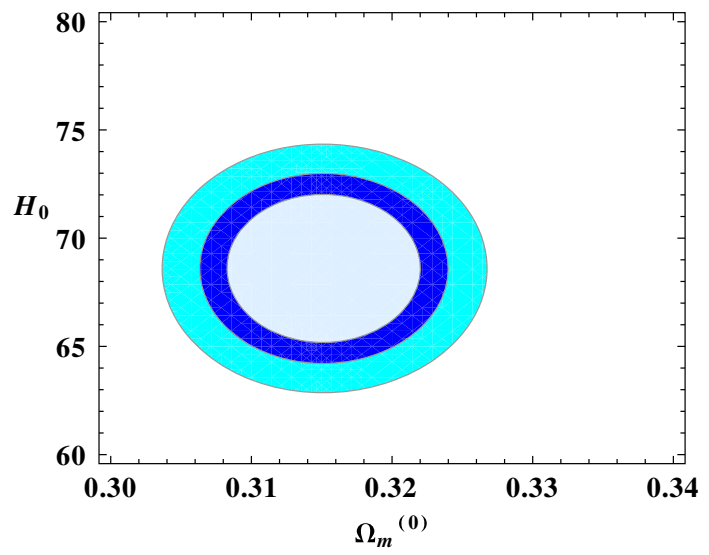

Fig. 7 Observational constraints on the parameters space $\left(\Omega_{m}^{(0)}-H_{0}\right)$ at the $68.3 \%$ (light blue), $90 \%$ (dark blue) and $99 \%$ (cyan) confidence levels

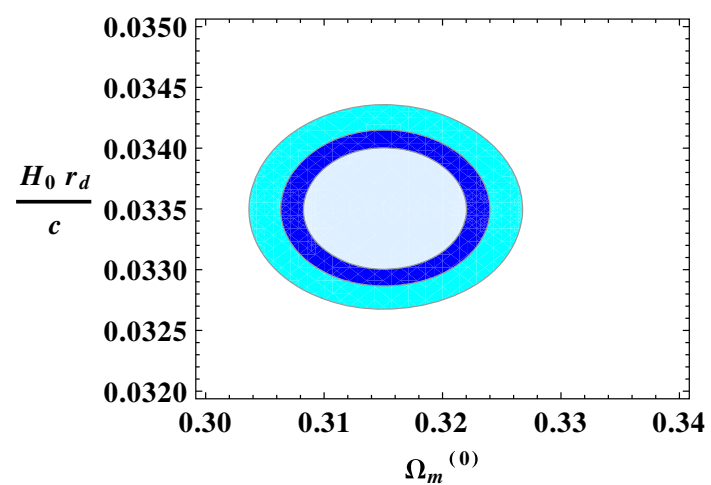

Fig. 8 Observational constraints on the parameters space $\left(\Omega_{m}^{(0)}-\right.$ $H_{0} r_{d} / c$ ) at the $68.3 \%$ (light blue), $90 \%$ (dark blue) and $99 \%$ (cyan) confidence levels

With a total of 1091 data points from SNe Ia, OHD, BAO and $\mathrm{CMB}$, we find from our data analysis a chi-square per degrees of freedom to be around 0.983 i.e., very close to 1 which in turn refelects the fact that the fitment of the model parameters are in good agreement with the observational data sets [78].

\section{Discussions and conclusions}

The present work shows that the dark energy can have its origin in the particle physics sector consistent with the observations coming from particle physics, such as Higgs vev and the dimensionless coupling constant arising from the $\mathrm{SU}(2)$ gauge coupling constant and the quartic coupling constant of Higgs field, as well as from the cosmological observations such as $\mathrm{SNe} \mathrm{Ia}$, OHD, BAO, CMB shift parameter data. As the masses of the elementary particles are generated via the interactions with the Higgs field, it is assumed that the Higgs field is isotropically and homogeneously present in the universe. However the Mexican hat potential of Higgs field is not flat enough to lead to the recent cosmic acceleration. The presence of SU(2) gauge field enhances the Hubble friction as well as generates an effective flat enough potential so that the Higgs dark energy can give rise to late time cosmic acceleration. The model turns out to be a viable dark energy model having its origin in the standard model of elementary particles so far as the observations are concerned. This is worth mentioning here that spacetime symmetries of FLRW metric leaves us with the only degree of freedom $f(t)$ allowed from the $\mathrm{SU}(2)$ gauge sector. From the analysis it is also evident that an effective potential for gauge field arises due to the interaction with the Higgs field. However the effective potential for the gauge degrees of freedom is minimum at $f=0$ thereby leading to the vanishing vacuum expectation value

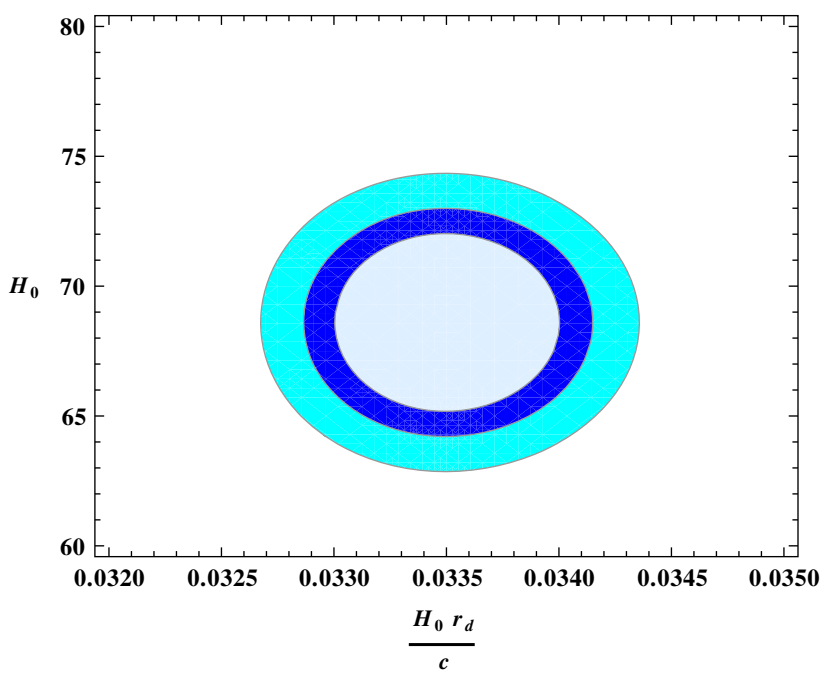

Fig. 9 Observational constraints on the parameters space $\left(H_{0} r_{d} / c-\right.$ $H_{0}$ ) at the $68.3 \%$ (light blue), $90 \%$ (dark blue) and $99 \%$ (cyan) confidence levels

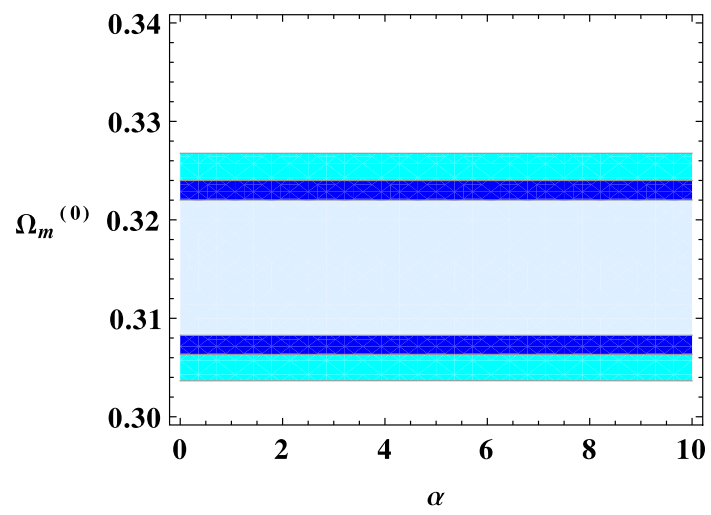

Fig. 10 Observational constraints on the parameters space $\left(\alpha-\Omega_{m}^{(0)}\right)$ at the $68.3 \%$ (light blue), $90 \%$ (dark blue) and $99 \%$ (cyan) confidence levels 
to the gauge fields in this theory [50] which is completely in agreement with the usual assumptions for the gauge fields in standard model of particle physics.

In this work, we study Higgs dark energy model in presence of gauge field in light of observational data from supernovae type Ia, baryon acoustic oscillation, observational Hubble data and cosmic microwave background shift parameter data. In performing the data analysis, we considered the initial conditions at the present epoch for dynamical evolution of the autonomous system. The choice of initial condition is in consideration with the vacuum expectation value of Higgs $v \sim 246 \mathrm{GeV}$, radiation density parameter at the present epoch of the order $10^{-4}$ and the total length of the radiation dominated epoch that leads to initial values of $x_{1}=10^{-18}, y_{1}=10^{-18}, z_{1}=10^{-18}, x_{2}=10^{-18}$, $y_{2}=0.831, w_{1}=10^{2}$ and $r=10^{-2}$ at $z=0$ i.e., present epoch $[49,50]$. These choice of initial conditions lead to correct cosmological dynamics for the observational universe as evident from Fig. 4 and the cosmic acceleration is a recent phenomenon. Moreover from the same figure it appears that the Higgs dark energy starts mimicking cosmological constant well in the radiation dominated era. The chi-square analysis of the observational data significantly constrains the model parameters $\left(\alpha, \Omega_{m}^{(0)}, H_{0}, H_{0} r_{d} / c\right)$. The minimum combined chi-square for all the data sets is obtained at the parameter values $\left(\Omega_{m}^{(0)}, H_{0}, H_{0} r_{d} / c\right) \sim(0.315,68.6$, 0.0335). Hence the sound horizon at the redshift of drag epoch turns out to be around $146.5 \mathrm{Mpc}$ which is in remarkably good agreement with the Planck 2018 results [8]. Also this is worth mentioning here that the chi-square per degrees of freedom is slightly greater than 0.98 which is the indication of a good fitting of the model with the observational data [78]. However data is still unable to provide any constraint on the model parameter $\alpha$ as is evident from Fig. 10. Needless to mention that the Higgs or the gauge field in the theory being minimally coupled to gravity does not conflict with the observational evidences of gravitational wave detection $[19,20,23]$. Thus the Higgs field in presence of gauge field turns out to be a viable candidate for dark energy so far as the observational data are concerned.

Data Availability Statement This manuscript has associated data in a data repository. [Authors' comment: All the data used in this article are available in the article except for the Pantheon Sample SNe Ia data points which are available at http://archive.stsci.edu/doi/resolve/resolve.html? doi=10.17909/T95Q4X.]

Open Access This article is licensed under a Creative Commons Attribution 4.0 International License, which permits use, sharing, adaptation, distribution and reproduction in any medium or format, as long as you give appropriate credit to the original author(s) and the source, provide a link to the Creative Commons licence, and indicate if changes were made. The images or other third party material in this article are included in the article's Creative Commons licence, unless indicated otherwise in a credit line to the material. If material is not included in the article's Creative Commons licence and your intended use is not permitted by statutory regulation or exceeds the permitted use, you will need to obtain permission directly from the copyright holder. To view a copy of this licence, visit http://creativecomm ons.org/licenses/by/4.0/.

Funded by $\mathrm{SCOAP}^{3}$.

\section{References}

1. A.G. Riess et al., Astron. J. 116, 1009 (1998). https://doi.org/10. 1086/300499

2. S. Perlmutter et al., Astrophys. J. 517, 565 (1999). https://doi.org/ $10.1086 / 307221$

3. R.R. Caldwell, M. Kamionkowski, Annu. Rev. Nucl. Part. Sci. 59, 397 (2009). https://doi.org/10.1146/ annurev-nucl-010709-151330

4. J. Frieman, M. Turner, D. Huterer, Annu. Rev. Astron. Astrophys. 46, 385 (2008). https://doi.org/10.1146/annurev.astro.46.060407. 145243

5. P.J.E. Peebles, B. Ratra, Rev. Mod. Phys. 75, 559 (2003). https:// doi.org/10.1103/RevModPhys.75.559

6. E.V. Linder, Rep. Prog. Phys. 71(5), 056901 (2008). https://doi. org/10.1088/0034-4885/71/5/056901

7. K. Bamba, S. Capozziello, S. Nojiri, S.D. Odintsov, Astrophys. Space Sci. 342, 155 (2012). https://doi.org/10.1007/ s10509-012-1181-8

8. N. Aghanim et al., Astron. Astrophys. 641, A6 (2020). https://doi. org/10.1051/0004-6361/201833910

9. L. Amendola, S. Tsujikawa, Dark Energy: Theory and Observations (Cambridge University Press, Cambridge, 2010). https:// books.google.co.in/books?id=Xge0hg_AIIYC

10. R.R. Caldwell, R. Dave, P.J. Steinhardt, Phys. Rev. Lett. 80, 1582 (1998). https://doi.org/10.1103/PhysRevLett.80.1582

11. P.J. Steinhardt, L. Wang, I. Zlatev, Phys. Rev. D 59, 123504 (1999). https://doi.org/10.1103/PhysRevD.59.123504

12. S. Capozziello, Int. J. Mod. Phys. D 11, 483 (2002). https://doi. org/10.1142/S0218271802002025

13. S. Capozziello, S. Carloni, A. Troisi, Recent Res. Dev. Astron. Astrophys. 1, 625 (2003)

14. S. Capozziello, V. Cardone, S. Carloni, A. Troisi, Int. J. Mod. Phys. D 12, 1969 (2003). https://doi.org/10.1142/S0218271803004407

15. A. Nicolis, R. Rattazzi, E. Trincherini, Phys. Rev. D 79, 064036 (2009). https://doi.org/10.1103/PhysRevD.79.064036

16. A. De Felice, S. Tsujikawa, Phys. Rev. Lett. 105, 111301 (2010). https://doi.org/10.1103/PhysRevLett.105.111301

17. T. Clifton, P.G. Ferreira, A. Padilla, C. Skordis, Phys. Rep. 513, 1 (2012). https://doi.org/10.1016/j.physrep.2012.01.001

18. S. Nojiri, S. Odintsov, V. Oikonomou, Phys. Rep. 692, 1 (2017). https://doi.org/10.1016/j.physrep.2017.06.001

19. B. Abbott et al., Phys. Rev. Lett. 119(16), 161101 (2017). https:// doi.org/10.1103/PhysRevLett.119.161101

20. A. Goldstein, P. Veres, E. Burns, M.S. Briggs, R. Hamburg, D. Kocevski, C.A. Wilson-Hodge, R.D. Preece, S. Poolakkil, O.J. Roberts, C.M. Hui, V. Connaughton, J. Racusin, A. von Kienlin, T.D. Canton, N. Christensen, T. Littenberg, K. Siellez, L. Blackburn, J. Broida, E. Bissaldi, W.H. Cleveland, M.H. Gibby, M.M. Giles, R.M. Kippen, S. McBreen, J. McEnery, C.A. Meegan, W.S. Paciesas, M. Stanbro, Astrophys. J. 848(2), L14 (2017). https://doi. org/10.3847/2041-8213/aa8f41

21. V. Savchenko, C. Ferrigno, E. Kuulkers, A. Bazzano, E. Bozzo, S. Brandt, J. Chenevez, T.J.L. Courvoisier, R. Diehl, A. Domingo, L. Hanlon, E. Jourdain, A. von Kienlin, P. Laurent, F. Lebrun, A. Lutovinov, A. Martin-Carrillo, S. Mereghetti, L. Natalucci, J. Rodi, J.P. Roques, R. Sunyaev, P. Ubertini, Astrophys. J. 848(2), L15 (2017). https://doi.org/10.3847/2041-8213/aa8f94 
22. B. Abbott et al., Astrophys. J. Lett. 848(2), L12 (2017). https://doi. org/10.3847/2041-8213/aa91c9

23. B. Abbott et al., Astrophys. J. Lett. 848(2), L13 (2017). https://doi. org/10.3847/2041-8213/aa920c

24. T. Baker, E. Bellini, P.G. Ferreira, M. Lagos, J. Noller, I. Sawicki, Phys. Rev. Lett. 119, 251301 (2017). https://doi.org/10.1103/ PhysRevLett.119.251301

25. P. Creminelli, F. Vernizzi, Phys. Rev. Lett. 119, 251302 (2017). https://doi.org/10.1103/PhysRevLett.119.251302

26. J. Sakstein, B. Jain, Phys. Rev. Lett. 119, 251303 (2017). https:// doi.org/10.1103/PhysRevLett.119.251303

27. J.M. Ezquiaga, M. Zumalacárregui, Phys. Rev. Lett. 119, 251304 (2017). https://doi.org/10.1103/PhysRevLett.119.251304

28. P. Creminelli, M. Lewandowski, G. Tambalo, F. Vernizzi, J. Cosmol. Astropart. Phys. 2018(12), 025 (2018). https://doi.org/10. 1088/1475-7516/2018/12/025

29. P. Creminelli, G. Tambalo, F. Vernizzi, V. Yingcharoenrat, J. Cosmol. Astropart. Phys. 2019(10), 072 (2019). https://doi.org/10. 1088/1475-7516/2019/10/072

30. J. Noller, Phys. Rev. D 101(6), 063524 (2020). https://doi.org/10. 1103/PhysRevD.101.063524

31. A. Casalino, M. Rinaldi, L. Sebastiani, S. Vagnozzi, Phys. Dark Univ. 22, 108 (2018). https://doi.org/10.1016/j.dark.2018.10.001

32. A. Casalino, M. Rinaldi, L. Sebastiani, S. Vagnozzi, Class. Quantum Gravity 36(1), 017001 (2019). https://doi.org/10.1088/ 1361-6382/aaf1fd

33. S. Weinberg, The Quantum Theory of Fields, vol. 2 (Cambridge University Press, Cambridge, 1996). https://doi.org/10. 1017/CBO9781139644174

34. A. Das, Lectures on Quantum Field Theory (World Scientific, Singapore, 2008). https://books.google.co.in/books? $\mathrm{id}=7 \mathrm{AX} 38 \mathrm{Cqeg} 3 \mathrm{kC}$

35. M. Henneaux, J. Math. Phys. 23, 830 (1982). https://doi.org/10. $1063 / 1.525434$

36. D. Galt'sov, M. Volkov, Phys. Lett. B 256(1), 17(1991). https://doi. org/10.1016/0370-2693(91)90211-8. http://www.sciencedirect. com/science/article/pii/0370269391902118

37. P. Moniz, J. Mourao, Class. Quantum Gravity 8, 1815 (1991). https://doi.org/10.1088/0264-9381/8/10/008

38. B. Darian, H. Kunzle, J. Math. Phys. 38, 4696 (1997). https://doi. org/10.1063/1.532116

39. J.D. Barrow, Y. Jin, K.I. Maeda, Phys. Rev. D 72, 103512 (2005). https://doi.org/10.1103/PhysRevD.72.103512

40. A. Banijamali, B. Fazlpour, Eur. Phys. J. C 71, 1684 (2011). https:// doi.org/10.1140/epjc/s10052-011-1684-4

41. E. Elizalde, A. Lopez-Revelles, S. Odintsov, S. Vernov, Phys. Atom. Nucl. 76, 996 (2013). https://doi.org/10.1134/ S1063778813080097

42. J. Cembranos, A. Maroto, S. Núñez Jareño, Phys. Rev. D 87(4), 043523 (2013). https://doi.org/10.1103/PhysRevD.87.043523

43. P.V. Moniz, J.M. Mourao, P.M. Sa, Class. Quantum Gravity 10(3), 517 (1993). https://doi.org/10.1088/0264-9381/10/3/012

44. U. Ochs, M. Sorg, Gen. Relativ. Gravit. 28, 1177 (1996). https:// doi.org/10.1007/BF02107381

45. A. Maleknejad, M. Sheikh-Jabbari, Phys. Lett. B 723, 224 (2013). https://doi.org/10.1016/j.physletb.2013.05.001

46. A. Maleknejad, M. Sheikh-Jabbari, J. Soda, Phys. Rep. 528, 161 (2013). https://doi.org/10.1016/j.physrep.2013.03.003

47. R. Namba, E. Dimastrogiovanni, M. Peloso, JCAP 11, 045 (2013). https://doi.org/10.1088/1475-7516/2013/11/045

48. F. Halzen, A.D. Martin, Quarks and Leptons: An Introductory Course in Modern Particle Physics (1984)

49. M. Rinaldi, Class. Quantum Gravity 32, 045002 (2015). https:// doi.org/10.1088/0264-9381/32/4/045002
50. M. Álvarez, J.B. Orjuela-Quintana, Y. Rodriguez, C.A. ValenzuelaToledo, Class. Quantum Gravity 36(19), 195004 (2019). https:// doi.org/10.1088/1361-6382/ab3775

51. J.A. Frieman et al., Astron. J. 135(1), 338 (2007). https://doi.org/ 10.1088/0004-6256/135/1/338

52. R. Kessler et al., Astrophys. J. Suppl. Ser. 185(1), 32 (2009). https:// doi.org/10.1088/0067-0049/185/1/32

53. M. Sako et al., Publ. Astron. Soc. Pac. 130(988), 064002 (2018). https://doi.org/10.1088/1538-3873/aab4e0

54. A. Conley, J. Guy, M. Sullivan, N. Regnault, P. Astier, C. Balland, S. Basa, R.G. Carlberg, D. Fouchez, D. Hardin, I.M. Hook, D.A. Howell, R. Pain, N. Palanque-Delabrouille, K.M. Perrett, C.J. Pritchet, J. Rich, V. Ruhlmann-Kleider, D. Balam, S. Baumont, R.S. Ellis, S. Fabbro, H.K. Fakhouri, N. Fourmanoit, S. GonzálezGaitán, M.L. Graham, M.J. Hudson, E. Hsiao, T. Kronborg, C. Lidman, A.M. Mourao, J.D. Neill, S. Perlmutter, P. Ripoche, N. Suzuki, E.S. Walker, Astrophys. J. Suppl. Ser. 192(1), 1 (2010). https://doi.org/10.1088/0067-0049/192/1/1

55. M. Sullivan et al., ApJ 737(2), 102 (2011). https://doi.org/10.1088/ 0004-637X/737/2/102

56. G. Miknaitis et al., Astrophys. J. 666(2), 674 (2007). https://doi. org/10.1086/519986

57. W.M. Wood-Vasey et al., Astrophys. J. 666(2), 694 (2007). https:// doi.org/10.1086/518642

58. G. Narayan et al., Astrophys. J. Suppl. Ser. 224(1), 3 (2016). https:// doi.org/10.3847/0067-0049/224/1/3

59. N. Suzuki, D. Rubin, C. Lidman, G. Aldering, R. Amanullah et al., Astrophys. J. 746, 85 (2012). https://doi.org/10.1088/0004-637X/ $746 / 1 / 85$

60. D.M. Scolnic, D.O. Jones, A. Rest, Y.C. Pan, R. Chornock, R.J. Foley, M.E. Huber, R. Kessler, G. Narayan, A.G. Riess, S. Rodney, E. Berger, D.J. Brout, P.J. Challis, M. Drout, D. Finkbeiner, R. Lunnan, R.P. Kirshner, N.E. Sanders, E. Schlafly, S. Smartt, C.W. Stubbs, J. Tonry, W.M. Wood-Vasey, M. Foley, J. Hand, E. Johnson, W.S. Burgett, K.C. Chambers, P.W. Draper, K.W. Hodapp, N. Kaiser, R.P. Kudritzki, E.A. Magnier, N. Metcalfe, F. Bresolin, E. Gall, R. Kotak, M. McCrum, K.W. Smith, ApJ 859(2), 101 (2018). https://doi.org/10.3847/1538-4357/aab9bb

61. R. Lazkoz, S. Nesseris, L. Perivolaropoulos, J. Cosmol. Astropart. Phys. 11, 010 (2005). https://doi.org/10.1088/1475-7516/2005/ $11 / 010$

62. P. Ade et al., Astron. Astrophys. 571, A16 (2014). https://doi.org/ 10.1051/0004-6361/201321591

63. C. Zhang, H. Zhang, S. Yuan, S. Liu, T.J. Zhang, Y.C. Sun, Res. Astron. Astrophys. 14(10), 1221-1233 (2014). https://doi.org/10. 1088/1674-4527/14/10/002

64. J. Simon, L. Verde, R. Jimenez, Phys. Rev. D 71, 123001 (2005). https://doi.org/10.1103/PhysRevD.71.123001

65. M. Moresco, A. Cimatti, R. Jimenez, L. Pozzetti, G. Zamorani, M. Bolzonella, J. Dunlop, F. Lamareille, M. Mignoli, H. Pearce, P. Rosati, D. Stern, L. Verde, E. Zucca, C.M. Carollo, T. Contini, J.P. Kneib, O. Le Fèvre, S.J. Lilly, V. Mainieri, A. Renzini, M. Scodeggio, I. Balestra, R. Gobat, R. McLure, S. Bardelli, A. Bongiorno, K. Caputi, O. Cucciati, S. de la Torre, L. de Ravel, P. Franzetti, B. Garilli, A. Iovino, P. Kampczyk, C. Knobel, K. Kovač, J.F. Le Borgne, V. Le Brun, C. Maier, R. Pelló, Y. Peng, E. PerezMontero, V. Presotto, J.D. Silverman, M. Tanaka, L.A.M. Tasca, L. Tresse, D. Vergani, O. Almaini, L. Barnes, R. Bordoloi, E. Bradshaw, A. Cappi, R. Chuter, M. Cirasuolo, G. Coppa, C. Diener, S. Foucaud, W. Hartley, M. Kamionkowski, A.M. Koekemoer, C. López-Sanjuan, H.J. McCracken, P. Nair, P. Oesch, A. Stanford, N. Welikala, J. Cosmol. Astropart. Phys. 2012(8), 006 (2012). https:// doi.org/10.1088/1475-7516/2012/08/006

66. M. Moresco, L. Pozzetti, A. Cimatti, R. Jimenez, C. Maraston, L. Verde, D. Thomas, A. Citro, R. Tojeiro, D. Wilkinson, J. 
Cosmol. Astropart. Phys. 05, 014 (2016). https://doi.org/10.1088/ 1475-7516/2016/05/014

67. A. Ratsimbazafy, S. Loubser, S. Crawford, C. Cress, B. Bassett, R. Nichol, P. Väisänen, Mon. Not. R. Astron. Soc. 467(3), 3239 (2017). https://doi.org/10.1093/mnras/stx301

68. D. Stern, R. Jimenez, L. Verde, M. Kamionkowski, S.A. Stanford, J. Cosmol. Astropart. Phys. 2010(2), 008 (2010). https://doi.org/ $10.1088 / 1475-7516 / 2010 / 02 / 008$

69. M. Moresco, Mon. Not. R. Astron. Soc. 450(1), L16 (2015). https:// doi.org/10.1093/mnrasl/slv037

70. Y. Yang, Y. Gong, J. Cosmol. Astropart. Phys. 06, 059 (2020). https://doi.org/10.1088/1475-7516/2020/06/059

71. J. Evslin, A.A. Sen, Ruchika, Phys. Rev. D 97(10), 103511 (2018). https://doi.org/10.1103/PhysRevD.97.103511

72. F. Beutler, C. Blake, M. Colless, D.H. Jones, L. Staveley-Smith et al., Mon. Not. R. Astron. Soc. 416, 3017 (2011). https://doi.org/ 10.1111/j.1365-2966.2011.19250.x
73. A.J. Ross, L. Samushia, C. Howlett, W.J. Percival, A. Burden, M. Manera, Mon. Not. R. Astron. Soc. 449(1), 835 (2015). https://doi. org/10.1093/mnras/stv154

74. M. Ata et al., Mon. Not. R. Astron. Soc. 473(4), 4773 (2018). https://doi.org/10.1093/mnras/stx2630

75. S. Alam et al., Mon. Not. R. Astron. Soc. 470(3), 2617 (2017). https://doi.org/10.1093/mnras/stx721

76. H. du Mas des Bourboux et al., Astron. Astrophys. 608, A130 (2017). https://doi.org/10.1051/0004-6361/201731731

77. J. Bayron Orjuela-Quintana, M. Álvarez, C.A. Valenzuela-Toledo, Y. Rodríguez, J. Cosmol. Astropart. Phys. (2020). https://doi.org/ 10.1088/1475-7516/2020/10/019

78. P.R. Bevington, D.K. Robinson, Data Reduction and Error Analysis for the Physical Sciences, 3rd edn. (McGraw-Hill, New York, 2003). https://cds.cern.ch/record/1305448 